\title{
Optics in Mexico
}

\author{
Zbigniew Jaroszewicz, ${ }^{* 1}$ Eduardo Tepichín-Rodriguez, ${ }^{2}$ and Jorge Ojeda-Castañeda ${ }^{3}$ \\ ${ }^{1}$ Institute of Applied Optics, Kamionkowska 18, 02-805 Warsaw, Poland \\ ${ }^{2}$ Instituto Nacional de Astrofisica, Optica y Electrónica, 72000 Puebla, México \\ ${ }^{3}$ Electronics Department, Campus Salamanca, University of Guanajuato, 36885 Guanajuato, México
}

Received March 31, 2015; accepted March 31, 2015; published March 31, 2015

\begin{abstract}
This editorial contains the overview of the contents of the current issue of Photonics Letters of Poland devoted to optics in Mexico.
\end{abstract}

It is a great honor and pleasure to have this opportunity to present the readers of Photonics Letters of Poland a new special issue of our journal prepared with the intention to show an animated, intense, and polyphacetic state-of-the-art of the science of optics in Mexico. It seems legitimate to start with a few words about not so much the history of optics in Mexico, because it would be too big topic as for this brief introduction, but more modestly, the cooperation in optics between Mexico and Poland.

Surprisingly, this cooperation is very vivid despite the large distance between the two countries. On the other hand it is not always well known. Maybe as a first if not evidence then at least its first forecast it could be acknowledged a book of the writer of Polish roots Elena Poniatowska The skin of the sky relating the beginnings of INAOE, The National Institute of Astrophysics, Optics and Electronics (in Spanish: Instituto Nacional de Astrofísica, Óptica y Electrónica), one of leading science research institutes in Mexico active in the area of optics. Another item worth mentioning is the book of Daniel Malacara Optical shop testing - surely everybody in Poland starting her/his adventure with optics was beginning with this source. The scientific collaboration can also demonstrate a long record of publications, starting from common papers of Prof. Krzysztof Patorski with Prof. Alejandro Cornejo-Rodriguez, going through common works of Dr. Piotr Szwaykowski with one of editors of this special number, Prof. Jorge OjedaCastañeda and with Prof. Victor Arrizon. A collaboration between Prof. Sabino Chávez-Cerda and Robert Iskander is another event worth mentioning. Another line of cooperation was established by Prof. Małgorzata Kujawińska together with Prof. Daniel Malacara.

More recent proof of Mexican-Polish collaboration is paper written to this special issue by Juan Carranza together with Konstantinos Falaggis and Tomasz Kozacki. In this paper there is determined a solution for the

\footnotetext{
*E-mail: mmtzjaroszewicz@post.pl
}

boundary problem for phase retrieval techniques based on the Transport of Intensity Equation. The whole issue contains 10 papers. Remaining papers present in this special number (except the last one) are a kind of the visit card showing a diversity of the Mexican optics. Kurt Bernardo Wolf reviews analogies between optical models with the same underlying symmetry group, what makes possible to apply similar approach in seemingly different context. Jorge Ojeda-Castañeda in one of his papers shows, how to design and use a pair of phase masks, which have both radial and helical variations, for optically implementing wavefront aberration generator. In his next paper, a similar idea is assumed by using two grey level masks for controlling the damping factor of an apodizer. In the subsequent work written together with Cristina Margarita Gomez-Sarabia another topic is addressed, namely the first order design of four optical devices, which use varifocal lenses for implementing optical systems. Fourth paper employs the Guigay coefficients for identifying a remarkably simple matrix treatment for describing first order dispersion of short pulses, which travel through a dispersion medium. In following publication Agustin Santiago-Alvarado et al. address recent advances in the development of tunable lenses which have produced more compact, simple and lightweight optical systems. Sukhoivanov, Ortiz et al. discuss a transformation of ultrashort optical pulses in a variety of optical fibers aiming synthesis of specially shaped pulses and single-pulse flat-top supercontinuum. Jesus Castañeda-Contreras et al. report a novel method to stabilize $\mathrm{Er}_{2} \mathrm{O}_{3}$ nanoparticles with ascorbate ligands. The $\mathrm{Er}_{2} \mathrm{O}_{3}$ nanoparticles had strong green and red emissions with sharp and well defined emission peaks. In addition, less intense UV, blue and NIR emissions were also detected. The luminescence was recorded under dynamical pumping at $525 \mathrm{~nm}, 805 \mathrm{~nm}$, and $975 \mathrm{~nm}$.

We hope that with this special issue we contribute into development of collaboration between Mexico and Poland and who knows, maybe to creation in the near future another special issue filled up in whole with joint papers written by Mexican and Polish authors. 